\title{
DOS CASOS DE TINEA NIGRA PALMARIS EN CIEGO DE AVILA (CUBA)
}

\author{
(Two cases of tinea nigra palmaris in Ciego de Avila(Cuba) \\ *Alfredo Estrada, O., *Pedro Morell,M., \\ *Miguel Suarez,H., **Yolanda Perez Jimenez. \\ *Hospital Provincial "Antonio Luaces Iraola" \\ **Centro Provincial de Higiene y Epidemiología. \\ Ciego de Avila ,CUBA.
}

Palabras clave: Tinea nigra, Phaeoannellomyces werneckii

Key words: Tinea nigra, Phaeoannellomyces werneckii

\section{RESUMEN}

Se presentan 2 casos de Tineanigra palmaris, uno no relacionado con ninguna fivente de contagio determinada y el otro con posibles adquisición desde el medio ambiente.

Se describen los datos clinicos y micológicos, identificandose al agente micótico causal como Phaeoannellomyces werneckii.

Se comenta la poc a frecuencia de esta afección en el pais, los casos reportados con anterioridad, la terapia empleada v la efectivarespuesta de los antimicóticos administrados:

\section{INTRODUCCION}

La tinea negra palmaris es producida por Phacoannellomyces werneckii (= Exophiala werneckii, = Cladosporium werneckii), el cual se ha diagnosticado en diversos paises de America. entre ellos USA, Puerto Rico, Panamá, Mexico. Costa Rica, Brasil y Venezuela $(1,2)$, mientras en Cuba se han publicado pocos casos $(3,4)$.

Se señala por algunos autores que el agente causal se distribuyeen el suelo y la vegetación en áreas geográficas tropicales y sub tropicales exclusivamente, e infecta al hombre mediante una solución de continuidad $(1,5)$.

La tinea nigra palmaris. se localiza generalmente en las eminencias tenar o hipotenar, así como en la concavidad de la mano, sin embargo, se han reportado localizaciones extra palmares. Las lesiones se caracterizan por manchas de color negro o pardo, las cuales se fusionan para formar placas policiclicas que tienen algunos centímetros de diámetro y cuỵo borde presenta una ligera

\section{SUMMARY}

Two case reports of tinea nigra palmaris are submitted here, one lacking any particular source of contagion and the other with probable signs of having become infected from the environment.

Clinical and mycological data are described while Phaeoanellomyces werneckii is identified as the causing mycotic agent. The little occurrence of this pathology in the country, the cases previously reported, the kind of therapy employed and the effective response of the antimycotic prescribed are also discussed.

elevación, no obstante, la superficie de la placa es lisa, no escamosa y generalmente apruriginosa. La lesión se atenúa o llega a desaparecer en ciertos enfermos con los lavados frecuentes de las manos $(1,6)$.

Nuestro objetivo principal, fue confirmar los 2 primeros casos reportados en la provincia de Ciego de Avila, Cuba.

\section{CASO CLINICO 1.}

Paciente de sexo masculino, de 56 años, raza blanca, atendido por consulta externa en el Policlínico de Especialidades.

El motivo de la consulta fue la presencia de manchas permanentes en la mano izquierda.

a) Historia de la enfermedad actual.

Paciente que presentaba una mancha oscura en la región palmar de la mano izquierda, desde hace aproximadamente 9 meses. La lesión fue interpretada por 
el paciente como suciedad, habiendo tratado de eliminarla con lavados frecuentes, sin resultado alguno. Inicialmente pequeña, pero debido a la aparición de otras manchas confluentes, adquiere un tamaño mayor.

El paciente no manifiesta prurito ni otra sensación subjetiva, no relacionando la adquisición del cuadro con ningún elemento determinado.

\section{b) Examen dermatológico.}

El cuadro cutáneo consistió en: máculas de color pardo oscuro, de tamaño variable, de bordes difusos, que confluyen formando una pequeña placa de 2 centímetros de diámetro, de superficie lisa y localizada en la región palmar de la mano izquierda, cerca de la región tenar.

\section{c) Examen micológico.}

1.- Examen directo: Luego de limpiar la zona con alcohol al 70\%, se practicó un raspado de la piel con bisturi estéril, colocando las escamas en una gota de dimetilsulfóxido, entre cubre y portaobjeto. La observa ción microscópica, reveló la presencia de hifas septadas, ramificadas y de color pardo negruzco.

2. - Cultivo. Parte de la descamación obtenida por el raspado fue sembrada en picadura, en cuatro tubos de Sabouraud glucosado, 2 con Actidiona $(0,5 \mathrm{~g} / \mathrm{l}) \mathrm{y}$ Cloranfenicol $(0,10 \mathrm{~g} / \mathrm{l})$, mientras los restantes con Penicilina y Estreptomicina $(20 \mathrm{U} / \mathrm{ml}, 40 \mathrm{U} / \mathrm{ml})$.

Los tubosfueron colocados a temperatura ambiente, durante 15 dias. Las colonias de Phueoannellomyces werneckii, inicialmente son de crecimiento lento, de tamaño restringido, de un color negro brillante, lisas , semejantes a colonias de levaduras y de aspecto mucoso. Con el tiempo adquirieron un micelio aéreo pardo verdoso.

Al microscópio, con tinción de "anilin-blue", se observaron predominantemente células levaduriformes gemantes, constituidas por blastoconidios ovales, hialinos, que con el tiempo se tornan oliváceos pálidos, ampliamente elipsoidales, unicelulares a bicelulares $6-9 \times 3,5-4,5 \mu \mathrm{m}$. Micelio con hifas anchas 4,5 a $6,5 \mu \mathrm{m}$, muy scptadas al madurar, de color café y de paredes gruesas, que exhibieron lateralmente, sobre células conidiógenas indiferenciadas, conspicuas zonas aneladas (anćlides).

3.- Diagnóstico micológico: Phaeoannellomyces werneckii (Horta) McGinnis \& Schell.1985

d) Tratamiento. Se indicú la aplicación tópica de pomada de Whitfield con azufre, sobre las lesiones 3 veces al día.

e) Evolución clínica. Al tercer día de iniciado el tratamiento, la lesión maculosa desaparece totalmente

\section{CASO CLINICO 2.}

Paciente de sexo femenino, de 11 años, raza blanca, atendida por consulta externa en el Policlínico de Especialidades.

El motivo de la consulta fue la presencia de manchas oscuras en la mano derecha.

\section{a) Historia de la enfermedad actual}

La madre refiere que desde hace varios meses comenzó en su hija, la aparición de una mancha oscura en la palma de la mano derecha que no desaparecia con los lavados frecuentes. Las lesiones no producian prurito ni otra sintomatología. Sin embargo, habiendo notado que en lugar de desaparecer, éstas habían aumentado de tamaño. decidió la consulta con un especialista. Debe destacarse que la niña solia jugar a menudo con hierbas y arbustos.

\section{b) Examen dermatológico.}

El cuadro clínico consistió en la presencia de placas de aproximadamente $3 \mathrm{~cm}$ de diámetro, formadas por máculas no solevantadas, de color pardo claro y oscuro, de tamaño variable (tomado la lesión un aspecto muy irregular).

c) Examen micológico y Tratamiento. Similar al caso 1 .

\section{DISCUSION}

En una casuística presentada en el estado de Zulia, Venczucla, se encontró cl predominio de la enfermedad en edades tempranas y medias de la vida, presentandose mayoritariamente en la raza blanca. En los niños se demostró un predominio de la localización en la mano derecha y en los adultos en la izquierda (7).

En nuestro estudio, la respuesta a la terapia fue rápida.

A pesar de lo escasos caso descritos en Cuba, nuestros hallazgos, permiten poner en conocimiento al facultativo, de la existencia de esta micosis, para evitar su posible confusión con un nevus pigmentado, el melanoderma de Addison's, o una hipercromía, cuya-conducta terapéutica sería mucho más dramática

La clasificación de los hongos dematiáceos semcjantes a levaduras, es a menudo compleja y controversial por su polimorfismo. Nishimura \& Miyaji (1984) (8) transfieren a Cladosporium werneckii a un nuevo género Hortaéa (Hortaea werneckii), sin embargo esta nueva denominación no es seguida por la mayoría de los micólogos(9).

McGinnis et. al., (1985) (10), proponen un nuevo 
nombre para ciertos hongos dematiáceos que producen células conidiógenas proliferativas percurrentes como Cladosporium werneckii, transfiriendolo a una nueva familia y nuevo género como Phaeoannellomyces werneckii (Horta) McGinnis \& Schell. Esta nueva denominación es más aceptada en la literatura actual.

\section{REFERENCIAS}

1.- Severo, L. C.; Bassanesi; M:C.; Londero,A.T.(1994). Tinea nigra: report of four cases observed in Ris Grande do Sul (Brazil) and a review of brazilian literature. Mycopathologia 126:157-162

2.- Rodriguez, J.; Gamboa, A.; Jaramillo, O.; Capra, J. (1988). Tinea nigra en Costa Rica. Presentación de ires casos clinicos por Phaeoannellomyces werneckii. Rev. Argent. Micol 11:21-31

3.- Armenteros, J.A. (1965). Micologia Médica. Editora Cientifica. La Habana, pp 80-85

4.-Cutlip, B.D. (1970). Tinea nigra Palmaris. Milit. Med 135: 192-193

5.- González, Q.; Ramírez, M.; Luis, B.; Belscr, T.; Edgar, E. (1988) Comportamiento de la Exophiala werneckii en medios de cultivo no tradicionales para hongos. Gac. Med. Caracas 96:393-39.1
6.- Ilayashi, M.; Kirgu, H.; Suerraga, Y.; Asahi, M. (1994). A case of cutaneous infection by Exophialu jeanselnsei. J. Dermatol .21: 971-973

7.-Vargas, II. (1986). Tinea nigra en el estado Zulia 1975-1985 (Venezuela). Dermatol. Venez. 24:143-146

8.- Nishimura, K. \& Miyaji,M.(1984). Hortaea, a new genus to accommodate Cladosporium werneckii. Japan. J. Med. Mycol. 25:139-146

9.- Hoog, G.S.de. \& Gerrits van Den Ende, A.H.C.(1992). Nutritional pattern and eco-physiology of Hortaea werneckii, agent of human tinea nigra. Antonie van Leeuwenhoek 62:321-329

10.- McGinnis,R.M.; Schell, A.WV.; Carson, J.(1985). Phaeoannellomyces and the Phaeococcomy'cetaceae, new dematiaceous blastomycetes taxa. J. Med. Vet. Mycol. 23:179-188 